\title{
CASE

\section{A Rare Case of Mullerian Adenosarcoma of Uterus in a 14-year-old Girl}

\author{
Mitra Mohit ${ }^{1}$, Minoo Saatian², Soheila Sarmadi ${ }^{3 凶}$
}

\begin{abstract}
'Department of Obstetrics and Gynecology, Bouali Hospital, Islamic Azad University, Tehran Medical Branch. ${ }^{2}$ Department of Pathology, Bouali Hospital, Islamic Azad University, Tehran Medical Branch.

${ }^{3}$ Department of Pathology, Women Hospital, Tehran University of Medical Sciences.
\end{abstract}

\begin{abstract}
Background: Mullerian Adenosarcoma, a rare tumor, is composed of mixed mesenchymal and epithelial elements. The epithelial component is typically benign and admixed with malignant Sarcomatous Stroma. This tumor can be distinguished in approximately $8 \%$ of all uterus sarcomas and commonly originates in the endometrium as a growing polypoid mass in the uterine cavity. All age groups are affected but the average age of uterine Adenosarcoma is about 58 . Case report: We aim to introduce a 14-year-old case of uterine Adenosarcoma as a rare occurrence, presenting with menometrorrhagia and formation of uterine fragile polypoid mass which extends into vagina. Total Abdominal Hysterectomy (TAH) and staging were performed. She had been clinically disease-free in a 40-month post operation follow-up. Distant metastasis is a very rare phenomenon, albeit the necessity of long-term follow-up seems necessary.Conclusion: Data accumulation of individual cases with such unusual neoplasm is essential for further experience provision.[GMJ.2014;3(4):256-9]
\end{abstract}

Key Words: Radiography; Dental Student; Diagnosis; Errors

\section{Introduction}

$\mathrm{M}$ ullerian Adenosarcoma (MA), a variant of Mullerian mixed mesodermal tumor (MMMT) of the uterus, is a rare neoplasm. It is characterized by a benign epithelial and a malignant stromal components and is observed in $8 \%$ of uterine sarcomas [1,2]. Affected patients are of different ages, from 14 to 89 , with the median age of 58 at presentation [3]. Most of the cases are menopausal women; therefore, Adenosarcoma is not commonly identified in adolescence. Along with Fleming et al. report, Mullerian Adenosarcoma has been reported in only 16 adolescent girls. They have

\section{GMJ}

2013 Galen Medical Journal

Fax: +98 7312227091

PO Box 7461686688

Email:info@gmj.ir presented a 10-year-old female diagnosed with Mullerian Adenosarcoma arising from endocervix [4]. MA presents as a polypoid mass in the myometrium, endocervix, ovary, round ligament of the uterus and pelvic wall [4]. The most common presenting symptom is abnormal vaginal bleeding and the most common finding in physical examination is a protruding tissue through a dilated cervix. The minority of patients are detected by enlarged uterus or palpable pelvic mass [5]. Since MA of the uterus is really rare especially in early adolescence, there exists quite limited experiences regarding both diagnostic methods and

\footnotetext{
Correspondence to:

Soheila Sarmadi, Associste Professor of Pathology,

Department of Pathology, Women Hospital, Tehran

University of Medical Sciences

Telephone Number: (+98) 2188906767

Email Address: ssarmadi@sina.tums.ac.ir
} 
treatment options. Due to the absence of long term follow-up data, the biological potential of this tumor is still unknown. As a consequence of these reasons, we describe a case of MA arising from uterus in a young patient presenting as vaginal mass.

\section{Case Presentation}

A 14-year-old girl was admitted to Bouali Hospital, Islamic Azad University, Tehran Medical Branch due to a nine-month period of menometrorrhagia. She has history of menstruation for 2 years. Pelvic ultrasonography revealed a larger-than-nulliparous-sized uterus $(85 \times 60 \times 50 \mathrm{~mm})$ and the endometrial thickness of $7 \mathrm{~mm}$ with normal cervix and adnexae, eight month ago. Her past medical history was not contributory. She received multiple hormonal therapies with Progesterone, four cycles of low dose oral contraceptives and two cycles of Cyproterone compound, all of which were of no use. In the recent two months, her vaginal bleeding increased in amount associated with profuse malodor vaginal discharge. Pelvic examination under general anesthesia revealed a large fragile fleshy mass protruding into vagina down to hymen. Evaluation of the cervix and determination of mass origin was not possible. The latest ultrasonography showed a solid huge mass in the uterus extending to vagina.

Multiple vaginal mass biopsies were performed. Upon histological examination, mature cartilaginous islands without obvious features of malignancy along with multiple

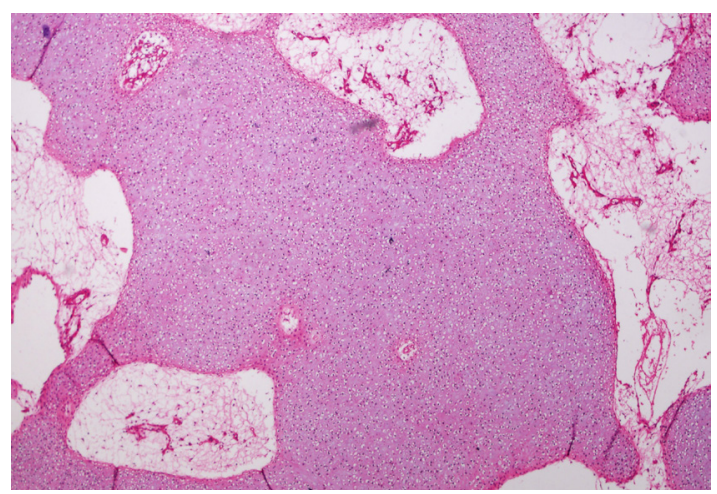

Figure 1. This Figure Shows Multiple Islands of Mature Cartilage Tissue. $(\times 40)$ inflamed necrotic tissues were reported in pathological description (Figure 1). Finally, total abdominal hysterectomy and staging including cytology, pelvic lymph node sampling and omentectomy were performed. Both ovaries were grossly normal. They were preserved and moved to the paracolic gutters up to the level of iliac crests and fixed to the underlying peritoneal surface.

Histopathology studies showed uterus was enlarged and occupied with a tumoral rubbery and grayish mass, measuring $16 \times 15 \times 3 \mathrm{~cm}$ which attached only to the uterine fundus. There was no evidence of tumoral extension to isthmus and cervix. Specimens were taken and processed for routine histological examinations. Hematoxilin-Eosin (H-E) staining revealed a neoplastic tissue composed of two epithelial and mesechymal elements. The epithelial elements were glandular, some cystically dilated lined by a single layer of columnar mucinous cells. Neither atypia nor mitosis was observed. Stromal component was unusually cellular consisting of atypical cells, periglandular cuffing, and more than three mitotic figures per 10 High Power Filed (HPF) (Figures 2,3). Myometrium and cervix were intact.

Although post-operative pelvic radiotherapy was indicated as a step in our therapeutic approach, the patient's family could not be convinced to accept any adjuvant treatment and follow up. Ten months later, she developed bilateral flank pain and gross hematurea. In physical examination, a huge fixed pelvic mass was detected. Pelvic and abdominal CT-

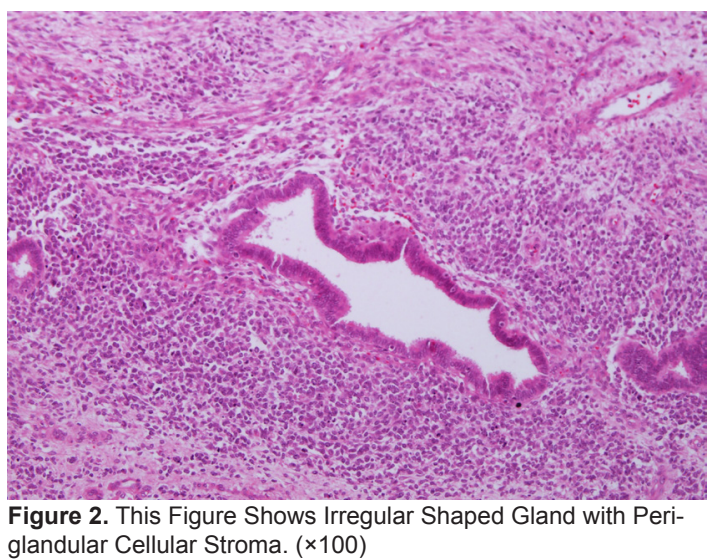

Figure 2. This Figure Shows Irregular Shaped Gland with Periglandular Cellular Stroma. $(\times 100)$ 


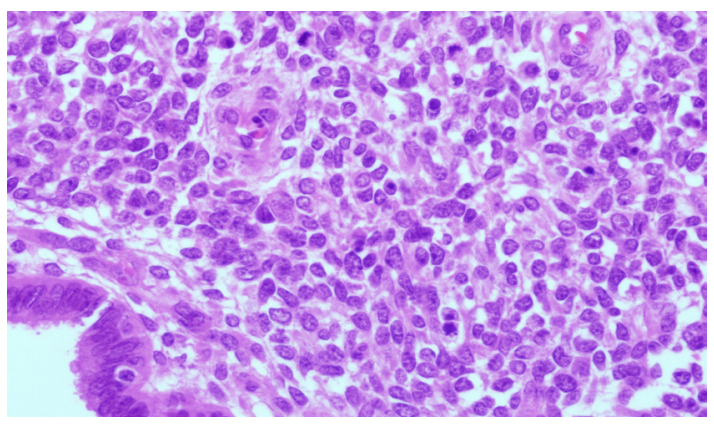

Figure 3. This Figure Shows Variable Stromal Cell Atypia and Stromal Mitotic Activity. $(\times 400)$

Scan revealed a large solid pelvic mass and bilateral hydronephrosis. Cystoscopy showed tumoral invasion to the posterior bladder wall. She received five cycles of Vincristin-Doxurubicin-Cyclophosphamide (CAV) regimen followed by four courses of Cyclophosphamide-Dactinomycin-Vincristin and six courses of Gecitabine-Docetaxel. At the end of chemotherapy, there was no evidence of tumor in pelvic examination, pelvis and abdominal MRI, cystoscopy and lung CT. Then, she received external pelvic irradiation $5000 \mathrm{CGY}$ in 25 fractions. The patient's consent to report the case was obtained orally in a Gynecologist-Oncologist office.

\section{Discussion}

Mullerian Adensarcoma belongs to a category of Mullerian mixed mesodermal tumors, which is infrequent among uterine malignancies, comprising benign glandular and malignant stromal components. It may be homologous (endometrial stroma, fibroblast and smooth muscle) or heterologous (cartilage, bone, etc.) [6]. MA was first described in 1974 by Clement and Scully. The slow growth rate of the tumor specifies it from the more aggressive forms of mixed mesodermal tumors and other uterine sarcomas [7]. The tumor mostly occurs within the uterine body in postmenopausal women [1]. Recognizing Adensarcoma in adolescents is not easily possible because of the infrequency of tumor at this age. Therefore, one of the striking things about our case was the age of clinical presentation. It is also worth considering that the main differential diagnosis of vaginal bleeding and vaginal pol- ypoid lesions at this age could be Embryonal Rhabdomyosarcoma (Sarcoma Botryoides). Embryonal Rhabdomyosarcoma, the most common malignant tumor of the vagina in infants and children, can occasionally present in cervix [8]. It consists of polypoid growth with densely-cellular submucosal cambium layer and scattered Rhabdomyoblasts. The stroma is edematous, not fibrous and the leaf-like pattern is absent. Immunohistochemical studies are helpful to demonstrate Rhabdomyoblastic differentiation in primitive tumor cells. Since in present case, we have characteristically epithelial component and also leaf-like pattern, immunohistochemical study was not performed.

Clement and Scully reported clinicopathologic analysis of 100 cases with uterine Adenosarcoma between the ages of 14 and 89 [2]. Most of the cases presented with abnormal vaginal bleeding as their symptom. They, like our case, revealed a large uterus with a polypoid mass in pelvic ultrasonography filling endometrium and protruding from the external ostium of the cervix. Adenosarcomas are considered as a low-grade malignant neoplasm with reported local recurrence of $25 \%$ [9]. Several unfavorable factors have been reported for MA including Sarcomatous overgrowth, high mitotic count, cytological atypia, heterologous elements, deep myometrial invasion, necrosis and extrauterine spread [8]. The presence of myometrial invasion and Sarcomatous overgrowth has been shown to be consistently associated with poor prognosis \& increased risk of recurrence $[8,6]$. Fortunately, except for the dominant presence of benign cartilaginous tissue (only observed in curettage specimen), there was no evidence of other poor prognostic factors in the present case. Optimal therapy for Adenosarcomas is still indeterminate. This uncertainty is mostly because this tumor appears in the early stages of reproductive life and its malignant potential is still poorly understood. Most authors recommend hysterectomy usually accompanied by bilateral Salpingo-Oopherectomy. However, case managing is quite challenging in an adolescent or a young nullipara for whom preserving fertility is of great importance $[8,10]$. For this patient, total abdominal hysterecto- 
my and staging were performed. Patients with superficial myometrial invasion of MA probably do not require radiation therapy, but those with more than a half invasion of the myometrium have a high likelihood of recurrence and might get more benefit from high-dose pelvic radiation with or without aggressive chemotherapy[1].

We decided to follow our patient with pelvic irradiation but the patient's family refused any adjuvant treatment and follow up. However, unfortunately ten months later, the tumor recurred. After that, she received both chemoand radiotherapy. She has been clinically well and disease-free in a follow-up period of 40 months.

Researchers reported an extremely rare case of MA of the uterus in a young girl. The optimal therapy for Adenosarcomas is still undefined. In our case, TAH with staging \& follow-up seemed to be an appropriate course of action. Long-term follow up is essential. Data accumulation of individual cases of such unusual tumors will indeed provide further experiences.

\section{References}

1. Ramos P, Ruiz A, Carabias E, Pinero

I, Garzon A, Alvarez I. Mullerian

Adenosarcoma of the cervix with heterologous elements: Report of a case and review of the literature. Gynecol oncol. 2002;84(1):161-66.

2. Tinar S, Sehirali S, Inal M, Yildrim Y, Celik E, Yigit S. Adenosarcoma of the uterus: a case report. Med Genl Med. 2004;6(1):51.

3. Fait T, Zivny J, Freitag P, Kuzel D.

Adenosarcoma of the uterine body in a 19year-old woman- three year survival: case report. Eur J Gynaecol Oncol. 2001;22(1):637.

4. Fleming NA, Hopkins L, de Nanassy J, Senterman M, Black AY. Mullerian Adenosarcoma of the cervix in a 10-year-old girl: case report and review of the literature. J Pediatr Adolesc Gynecol. 2009; 22(4):e4551.

5. Piura B, Rabinovich A, Meirovitz M, YanaiInbar I .Mullerian Adenosarcoma of the uterus: case report and review of literature. Eur J Gynecol Oncol. 2000;21(4):387-90.

6. Arend R, Bagaria M, Lewin SN, Sun X, Deutsch I, Burke WM, Herzog TJ, Wright
JD.Long-term outcome and natural history of uterine Adenosarcomas. Gynecol Oncol. 2010;119(2):305-8.

7. Park HM, Park MH, Kim YJ, Chun SH, Ahn JJ, Kim CI, et al . Mullerian Adenosarcoma with sarcomatous overgrowth of the cervix presenting as cervical polyp: a case report and review of the literature. Int J Gynecol Cancer. 2004;14(5):1024-9.

8. Manoharan M, Noor Azmi MA, Soosay G, Mould T, Weekes AR. Mullerian Adenosarcoma of uterine cervix: report of three cases and review of literature. Gynecol oncol. 2007;105(1):256-60.

9. Sinha A, Phukan JP, Sengupta S, Guha P. Mullerian Adenosarcoma of uterus with sarcomatous overgrowth and heterologous component associated with stromal deposit in omentum: a case report and review of the literature. Case Report Med. 2012;2012:820378.

10. Yu-Ping Xie • Hong-Xia Yao • Yang-Mei Shen. Müllerian Adenosarcoma of the uterus with heterologous elements: two case reports and literature review. Arch Gynecol Obstet. 2012;286(2):537-40. 